\title{
Research on the Interference Cancellation Based on Adaptive Algorithms
}

\author{
Jing Dai*, Zhonghua Han, Feng Zhang \\ Faculty of Information and Control Engineering, Shenyang JianZhu University, Shenyang, China \\ *Corresponding author's Email: daijing6615@163.com
}

\begin{abstract}
The adaptive interference cancellation algorithms(AICA) is proposed in this paper. The Matlab simulation results of the performance of six different kinds of AICA are presented. According to the comparison of the simulation results, especially the usage of resource, convergence rate and effects of cancellation, the NLMS adaptive interference cancellation algorithm and frequency-domain block LMS algorithm are selected, which are suitable for the further design on FPGA. By simulating the functions by Modelsim, and analyzing the PSD curve and the constellation of output signals, the adaptive interference cancellation algorithm system based on frequency-domain block LMS have achieved expected effect. The frequency-domain block LMS adaptive echo interference cancellation system has better flatness on the in-band PSD curve and better convergence effect on the constellation. Therefore, frequency-domain block LMS adaptive interference cancellation algorithm is chosen as the design with high performance-price ratio. The more points there are, the more obvious advantages it will perform.
\end{abstract}

Keywords: Adaptive algorithms; Interference cancellation; NLMS; Frequency-domain block LMS; FPGA

\section{Introduction}

In modern mobile communication systems, a mobile communication receiver should have a wider frequency range and real time signal processing capabilities in complex signal environment. An adaptive digital filter is the best option that can improve the receiver sensitivity under the condition of low signal to noise ratio (SNR). Adaptive filters can process data without any prior information about signal environment and the received signals. The study of adaptive digital filters with high-speed data processing capabilities is currently an important issue in signal processing fields [1]. In recent years, the rapid advancement in digital technologies has supported the implementation of sophisticated digital signal processing algorithms for real-time applications. Especially, with the development of FPGA (Field Program Gate Array), many signal processing algorithms can be implemented, which are theoretically reasonable but could not be implemented for the hardware design previ- ously. It is urgent that more complex, larger amount of high-speed data signals be processed on FPGA chip [2].

In mobile communication system, repeater plays an essential role in the coverage and optimization of networks. The weak received signals will first be amplified by a repeater, and then are emitted to potential coverage area by the transmitting antenna, so as to realize the extension and coverage of communication networks. However, due to the same-frequency transmitting characteristics, without taking effective measures, the signals emitted by the transmitting antenna will be caught by the receiving antenna from the same repeater some time later, which may lead to the strong interference to the effective signals, which is echo interference. The interference will have a bad influence on the communication. It may even cause the impossibility of communication in severe case. To deal with the problem of echo interference, traditional methods involve keeping some distance between the receiving antenna and the transmitting antenna when 
installing the repeater so as to effectively isolate signals. With the development of digital signal processing technology and large scale programmable devices, the adaptive echo interference cancellation technology has become the effective method to cancel echo interference in repeater systems. It can figure out the problem of spatial isolation of a repeater; meanwhile, it can greatly improve the quality of communication.

This study focuses on the WCDMA repeater, under the environment of MATLAB. It carries out the model building and simulation research on interference cancellation system on the basis of LMS (Least Mean Squares) adaptive filtering algorithm [1][3], NLMS (normalized LMS) adaptive filtering algorithm[4], symbolic LMS adaptive filtering algorithm, delay LMS adaptive filtering algorithm, frequency-domain block LMS adaptive filtering algorithm[5], and RLS (Recursive Least Squares) adaptive filtering algorithm. In the light of the easiness of hardware implementation and high-speed processing, comparisons are made between six kinds of adaptive filtering algorithm [6][7]. Finally, the NLMS adaptive filtering algorithm and the frequency-domain block LMS adaptive filtering algorithm are chosen for further hardware architecture design and programming realization. And, the performance of the achieved system is verified.

\section{Basic Principles of Adaptive Interference Cancellation}

Echo interference is the kind of time-varying, unknown and multipath interference. This characteristic requires the filter can automatically track the changes of signals, and respond to the changes by adjusting the weight coefficient quickly. What is more, this just fits well with the functions of the adaptive filter [2]. Thus, the adaptive filter is what to be chosen for the echo interference cancellation. The principles of adaptive filtering echo interference cancellation is shown in Figure 1.

In Figure 1, the signals emitted by the transmitting antenna will produce echo interference after multipath echo channel. The echo interference, superposed with the weak signals from the base station, enters into the receiving antenna and causes the echo interference to the repeater. The adaptive filtering algorithm uses the signals from the transmitting antenna as reference to adjust the weight coefficient of the filter so that the interference estimated value of echo channel is simulated by the filtering processing unit. Then the estimated value is subtracted from the receiving signals superposed with echo interference, thus the cancel- lation of echo interference is fulfilled. If only the transfer function of adaptive filter can fit well with the transfer function of the echo channel, reflecting the actual echo channel more truthfully, it will be possible to realize the complete cancellation of echo interference from receiving signals and get communication signals of high-quality. Therefore, the key of realizing echo interference cancellation is adaptive filter.

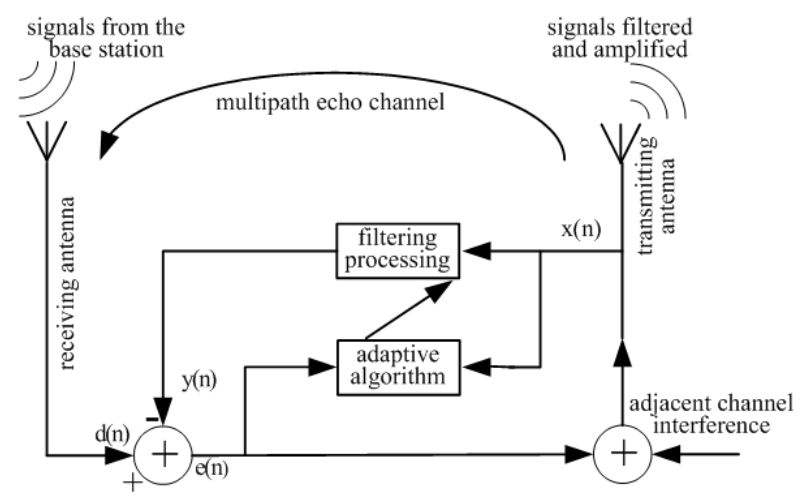

Figure 1 The principle diagram of echo interference cancellation of adaptive filter

An adaptive digital filter can filter the time variant interference signals which exist in the communication channels; thereby high quality communication signals can be obtained. Many researchers focus on the development of adaptive algorithm in mobile communication systems. Figure 2 shows the block diagram of an adaptive digital filter, where $d(n)$ is a desired signal, $y(n)$ filter's output signal, and error $e(n)$ adjusts the adaptive digital filter coefficients in a weight vector $\omega$.

In order to determine the optimal weight vectors, some algorithms have been studied. Among all the algorithms, Least Mean Square (LMS) is the most common. The others are the improvement based on the LMS algorithm.

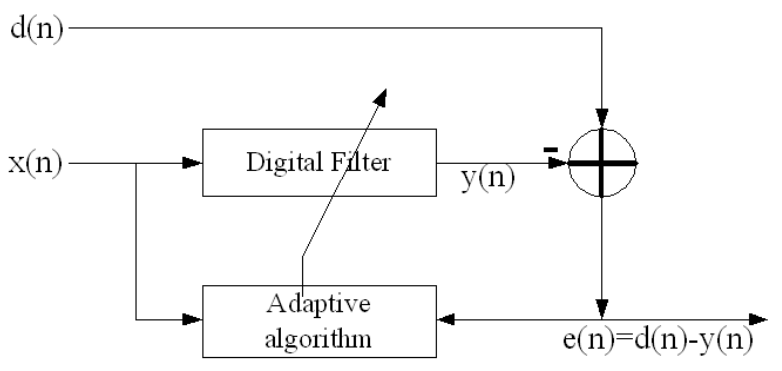

Figure 2 An adaptive filter block diagram 


\section{Adaptive Algorithm Analysis}

\subsection{LMS algorithm}

In 1960 Widrow and Hoff presented the LMS algorithm. LMS algorithm was studied based on Minimum Mean Square Error (MMSE) and the steepest descent algorithm. From LMS algorithm we know that

$$
y(n)=\sum_{i=1}^{n} \omega(i) x(n-i+1)=\omega^{T} X(n) .
$$

Where $y(n)$ is the output at time $n$. The input signal $x(n)$ is multiplied with the coefficients in a weight vector $\omega . \omega(n+1)$ is estimated according to the following recursion:

$$
\omega(n+1)=\omega(n)+\mu x(n) e^{*}(n),
$$

Where $\mu$ is a small positive constant, called the step size, which controls system stability and convergence rate. Because $\mu$ is a constant, convergence rate is slower, and misadjustment coefficient $\delta$ is still bigger when the system reaches steady state. $\delta$ could be expressed as:

$$
\delta=\mu M P_{\text {in }}
$$

$M$ is filter order, $P_{i n}$ is input signal power. As can be seen from Equation (3), when the step size $\mu$, input signal power $P_{i n}$, or filter order $M$ is large, the misadjustment coefficient $\delta$ is large. LMS algorithm can not achieve both rapid convergence and small steady-state error simultaneously.

\subsection{Normalized LMS algorithm}

Normalized LMS algorithm is an improved algorithm of LMS. NLMS algorithm will be easy to implement with faster convergence speed and accurate tracking capability [8]. Equation (3) shows misadjustment coefficient $\delta$ is constant if $\mu$ and $P_{i n}$ are inversely proportional to the change. Thus NLMS algorithm will be converged faster than LMS algorithm. $(n+1)$ is estimated according to the following simple recursion [8]:

$$
\begin{aligned}
& \omega(n+1)=\omega(n)+\frac{\hat{\mu}}{x^{H}(n) x(n)+\psi} e^{*}(n) x(n), \\
& \frac{\hat{\mu}}{x^{H}(n) x(n)+\psi}=\mu(n) .
\end{aligned}
$$

Where $\hat{\mu}$ is a small positive constant, called the step size, whose value is between 0 and 1 . A small constant $\psi$ is used to modify the algorithm to avoid the possible large step size which may result that the NLMS algorithm will be diverged when $x^{H}(n) x(n)$ is very small.

As can be seen from equation (5), the step size $\mu(n)$ varies adaptively by following the changes in the input signal level. This prevents the update weights from diverging and makes the algorithm more stable and faster converging than when a fixed step size is used.

Figure 3 shows the MSE (Mean square error) plot or convergence for the LMS and the NLMS algorithm respectively, which have the same filter orders. As shown in Figure 3, for the same adaptation size or iterations, the NLMS algorithm can achieve much faster convergence than the LMS algorithm.

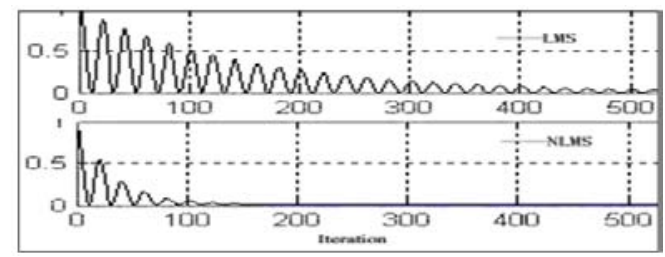

Figure 3 MSE plot for the LMS and NLMS algorithm respectively

\subsection{Recursive least squares algorithm}

The Recursive Least Squares (RLS) algorithm is based on the least square criterion; difference square sum is to be minimized between reference signals and output signals of filter as the ultimate objective. The iterative equation of filter can be expressed as equation (6).

$$
\omega(n)=\omega(n-1)+\frac{1}{\lambda} R^{-1}(n-1) e(n) x(n),
$$

where $0<\lambda \leq 1$ is genetic factor, $R(n-1)=E\{x(n-$ 1) $x^{T}(n-1)$ the autocorrelation matrix at time $n-1$ of input signals.

\subsection{Delay LMS algorithm}

Considering the slow convergence process, and the contradiction between step factor and convergence rate, Long and Herzberg proposed the delay LMS (DLMS) algorithm which is derived from the traditional LMS algorithm. Comparing with LMS algorithm, the delayed cycle number $\mathrm{d}$ in figure 4 used in updating the coefficients of DLMS algorithm is to generate the coefficient at the next clock. Thus, the throughput of DLMS algorithm data is twice as that of LMS, which is more suitable for the high-speed signal processing [9]. 


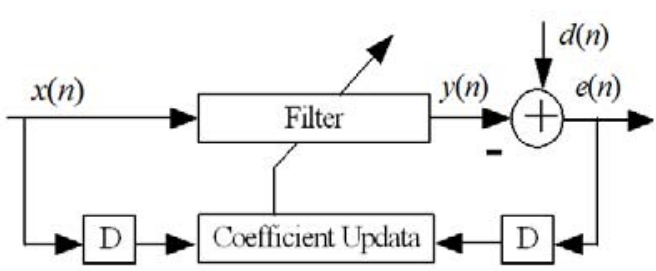

Figure 4 Block diagram of DLMS algorithm

The iterative equation of filter can be expressed as equation (7).

$$
\omega(n)=\omega(n-1)+2 \mu e(n-d) x(n-d),
$$

where the parameter $d$ is the delayed cycle number, which is introduced when the weight coefficients are updated.

\subsection{Sign LMS algorithm}

Stochastic gradient descent method is also used in Sign LMS algorithm in order to obtain the optimal solution. However, only optimal solution not the size of gradient is given in this method. The iterative equation of filter can be expressed as follows:

$$
\begin{aligned}
& \omega(n)=\omega(n-1)+2 \mu \cdot \operatorname{sign}[e(n)] x(n), \\
& \omega(n)=\omega(n-1)+2 \mu \cdot e(n) \operatorname{sign}[x(n)],
\end{aligned}
$$

where sign[] is sign function. Equation (8) is the Sign LMS algorithm of error signals and equation (9) the input signals.

\subsection{Frequency-domain block LMS algorithm}

The basic principle of the block LMS algorithm is to divide the input sequences $x(n)$ into the data blocks at the length of $\mathrm{L}$ point. The adaptive filter updates the weight coefficients block by block instead of one by one. The block LMS algorithm mainly concerns the liner convolution between the weight coefficients and input signals, and the linear correlation between the input signals and the error signals. And fast fourier transformation (FFT) is especially suitable for fast convolution and relative calculation. Thus the block LMS algorithm is conducted in frequency domain, or, Frequency-domain Block LMS.

\section{Adaptive Interference Cancellation System Simulation Comparison}

Figure 5 shows the simulation model block diagram, which indicates WCDMA source, echo interference cancellation block, Wiener Baseband Power Amplifier (PA), synchronization module and multipath channel. The signals from the WCDMA source are inputted to the system. Output signals from the echo cancellation block (Point $\mathrm{C}$ ) are amplified by the power amplifier and then fed back via multipath channel to the input terminal as interference signal. The interference signals mix with the WCDMA source signals and together enter the echo cancellation block. Meanwhile, the output signals of the power amplifier serve as the reference signal of the cancellation block (Point $\mathrm{G})$. The cancellation of echo interference is processed in the baseband. The original bandwidth of WCDMA source is $3.84 \mathrm{MHz}$, and the stopband bandwidth becomes $4.6848 \mathrm{MHz}$ via a shaping filter with roll-off factor 0.22 . The cancellation block adopts several algorithms, including LMS adaptive filtering algorithm, NLMS adaptive filtering algorithm, sign LMS adaptive filtering algorithm, DLMS adaptive filtering algorithm, frequency-domain block LMS adaptive filtering algorithm, and RLS adaptive filtering algorithm. In the simulation process, the multipath echo interference is three-path, and the order of the filter is 128 , and the step size of the simulation 0.00008 . For each kind of adaptive algorithm, echo interference cancellation simulation is implemented to input signals with the signal to interference ratio $-10,0,10$, respectively.

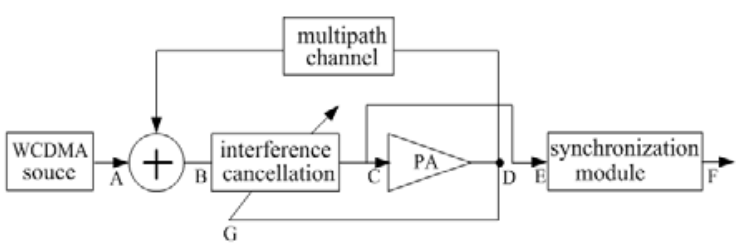

Figure 5 Adaptive echo interference cancellation system simulation model block diagram

In analyzing the PSD curve and the constellation of simulation results, it turns out that there is some improvement in the distribution on constellation for the sign LMS adaptive filtering algorithm. The output signals are not flat enough on the in-band PSD curve, which varies from the PSD curve of the source signals. Moreover, the attenuation of out-band does not reach the objective of $40 \mathrm{~dB}$ when the signal to interference ratio is -10. This shows that sign LMS adaptive filtering algorithm does perform some inhibiting ability to multipath echo interference. However, the effect is not satisfying.

While the other five sorts of adaptive filtering algorithm perform excellent echo cancellation ability judging from the PSD curve and the constellation of 
simulation results, and it can meet the design requirement of the echo cancellation system. In the view of the easiness to implementation on FPGA chips, this paper discusses the convergence rate and the usage of resource of five adaptive algorithms-LMS, NLMS, DLMS, frequency-domain block LMS and RLS, and the one that best fit for high-speed signal processing and implementation on FPGA is chosen.

As for the convergence rate, on the same condition of 128 order and 0.00008 step size, RLS has the highest convergence rate, followed by frequency-domain block LMS, NLMS, DLMS, and LMS in sequence. When the tap number of filter $\mathrm{M}$ is 512 , frequencydomain block LMS is 8.8 times faster than LMS, and 16 times faster when $\mathrm{M}$ is 1024 .

And the usage of resource: if the length of the data blocks to be processed is $M$, LMS will need to operate corresponsive $2 M^{2}$ times multiplication operation; sign LMS will need $M^{2}$ times multiplication operation; frequency-domain block LMS will need $10 M \times$ $\log _{2} M+26 M$ times multiplication operation; RLS will need $\left(2 M^{2}+4 M\right) M$ times multiplication operation and twice division operation; and NLMS will need multiplier little more than LMS when adopts optimization algorithm.

RLS has the highest convergence rate but huge usage of resource. Sign LMS has the least usage of resource, but it can not give satisfying filtering effect. LMS is too slow at the convergence to satisfy the requirement of the high-speed signal processing, though the usage of resource is relatively small and satisfying filtering effect. Therefore, this paper adopts NLMS adaptive algorithm and frequency-domain block LMS adaptive algorithm to design the adaptive echo interference cancellation system.

\section{NLMS Adaptive Algorithm Filter Imple- mentation on FPGA}

In this work, we considered a NLMS algorithm adaptive filter with 17 orders. And the filter has 16-bitwide input data and output data. The FPGA chips supported by Xilinx Company are the Virtex 5 series. The program was written by verilog HDL language. The Modelsim simulation software was used to run simulation for the designed system. The simulated results were carried out spectrum analysis under matlab environment. Equation (5) shows that $\mu(n)$ can be expressed by a division formula. Because the value of $\eta$ is between 0 and 1 , the product of $\mu(n)$ and $e(n)$ can be obtained by shift operation to reduce the computational complexity.
When the value range of equation $x^{H}(n) x(n)$ is judged, the number of left shifting bit was decided for the error $e(n)$, let $\eta=1 / 256, \psi=1$, then $\mu(n)_{\max }=$ $1 / 256=2^{-8}, \mu(n)_{\min }=1 / 2274877906945 \approx 2^{-38}$. Since the bit width of $e(n)$ is 32, in segmentation operation, when $x^{H}(n) x(n)=0, e(n)$ was shifted 8 bits to the left; when $1 \leq x^{H}(n) x(n) \leq 2, e(n)$ was shifted 9 bits to the left; when $3 \leq x^{H}(n) x(n) \leq 7, e(n)$ was shifted 10 bits to the left. And the rest may be known by analogy. When $x^{H}(n) x(n) \geq 4194303, e(n)$ will be shifted 31 bits to the left. According to segmentation and approximation method, shift operation was adopted instead of division. In addition, appropriate expansion and interception must be considered for every calculating result to prevent data overflow. NLMS algorithm adaptive filter structure is shown in Figure 6.

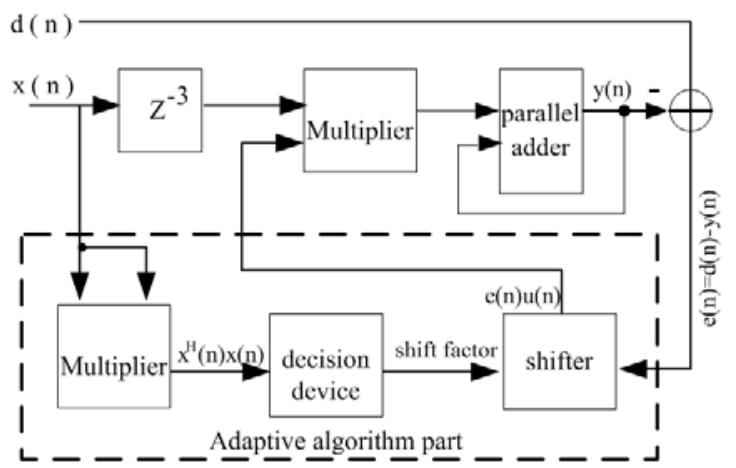

Figure 6 NLMS algorithm adaptive filter structure

In Figure 6, each block represents a computing node. The adaptive algorithm parts consist of multiplier, decision device, and shifter. The multiplier performs the operation of equation $x^{H}(n) x(n)$. The decision device which is based on case select structure chooses shift factor according to the different range of $x^{H}(n) x(n)$. The shifter implements shifting and interception for error $e(n)$. The multiplier, decision device, and shifter delays 1 clock period respectively, so $x(n)$ must be delayed 3 clock periods to avoid time sequential confusion. It reduces 17 clock periods and speeds up data processing that the multiplier, decision device, and shifter replace a divider. The parallel adder will adds up the weight coefficient of each tap to the corresponding product of $x(n)$. The function of $Z^{-3}$ will produce 3 clock periods of delay to input signal $x(n)$.

\section{Performance Analysis of the NLMS Adap- tive Echo Cancellation System}

With the help of verilog HDL hardware programming language, program for the NLMS adaptive filter algorithm, synthesize in the environment of ISE, and 

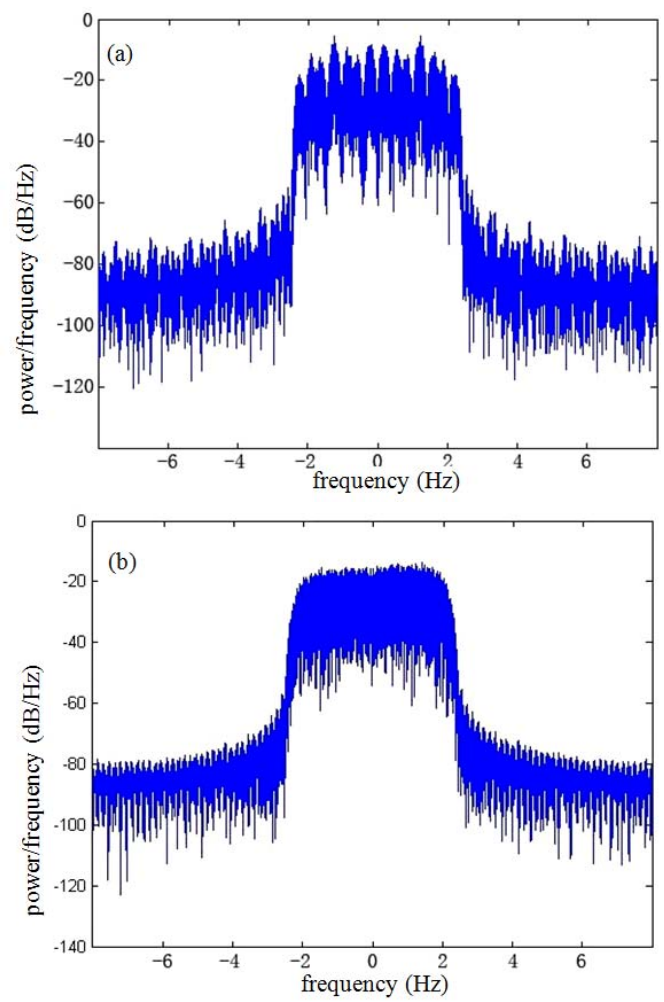

Figure 7 The Comparison between the PSD curves before and after the interference cancellation when the signal to interference ratio is -10 . (a). Before the interference cancellation (b). After the interference cancellation

achieve function simulation using Modelsim. Based on the adaptive algorithm above, echo interference cancellation was implemented to the signals with the signal to interference ratio $-10,0,10$ respectively. Then the output data from Modelsim were imported to Matlab. By analyzing the PSD curve and the constellation of the imported data, estimate the performance of the adaptive echo interference cancellation system [10].

Figure 7 and Figure 8 show the performance analysis of NLMS adaptive echo interference cancellation system when the signal to interference ratio is -10 . Before interference cancellation, the in-band PSD curve has wider fluctuation, and the distribution of the constellation is dispersal. While after interference cancellation, the in-band PSD curve becomes more flat and the out-band attenuation exceeds $40 \mathrm{~dB}$. This indicates that the NLMS adaptive echo interference cancellation system has remarkable inhibition ability to multipath echo interference.

\section{Frequency-Domain Block LMS Adaptive Algorithm Filter Implementation on FPGA}

Frequency-domain Block LMS adopts the 1/2 overlapping-reserving method in the operation process. The
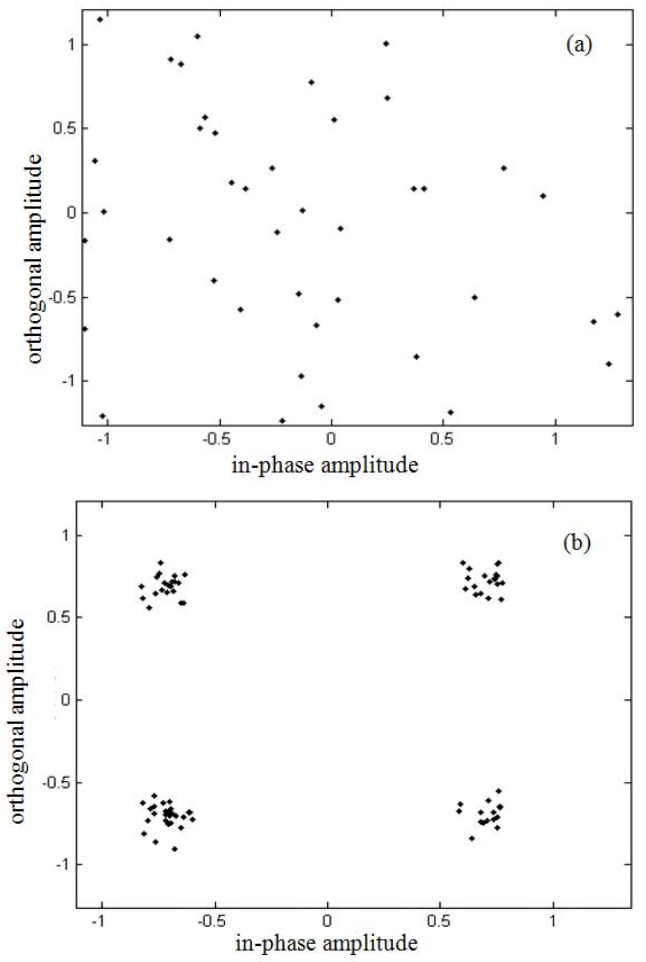

Figure 8 The comparison between the constellation before and after the interference cancellation when the signal to interference ratio is -10 . (a) Before the interference cancellation (b) After the interference cancellation

number of the coefficients equals to the size of the data block, so as to get the highest computational efficiency [11]. The frequency-domain block LMS algorithm based $1 / 2$ overlapping reserving method cascades the input signals and the expected signals, conducts FFT, and then operates multiplication in frequency domain, which needs to be operated with fast convolution and relative calculation in time domain [12]. The basic block diagram of algorithm is shown in Figure 9.

Figure 9 consists of 4 parts.

I ) Every $M$ input signals $x(n)$ in the time domain make up a block; every two cascaded data blocks conduct $N$ point fast fourier transformation, and get transferred $X(k)$ in the frequency domain. $X(k)$ is the input of the adaptive filter. $N$ is twice as much as $M$ - the number of the filter tap.

II ) Update the weight coefficient $W(k)$ according to the input signal $X(k)$ and error signal $E(k)$ and obtain the estimated value of interference $Y(k)$.

III ) Carry out inverse fast fourier transformation (IFFT) for the output signal of adaptive filter $Y(k)$, and get the time domain signals- interference estimated value $y(n)$.

IV ) Calculate the differences between the interfered signal $d(n)$ and $y(n)$, and get the signal $e(n)$ in which 


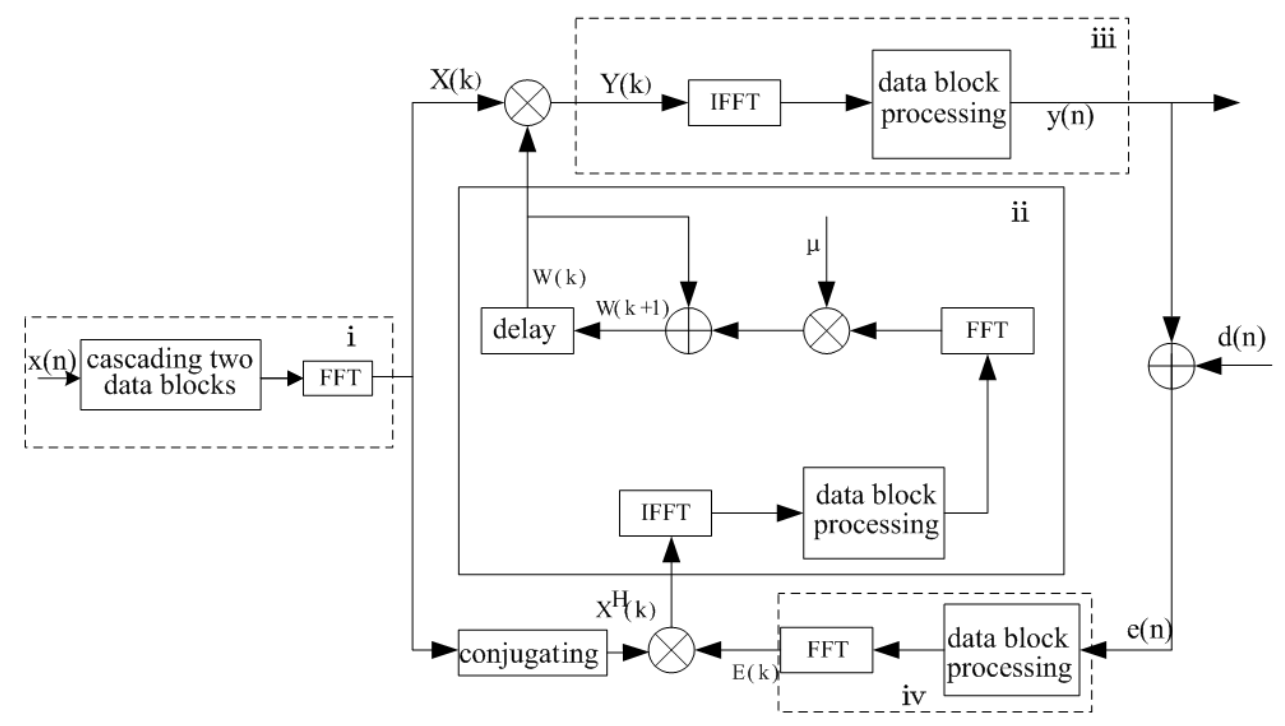

Figure 9 The basic block diagram of frequency domain block LMS algorithm

the interference is removed. Transforming the signal $e(n)$ by fourier transformation will get the frequency domain value $E(k)$, which can serve for generation filter tap coefficient of the next iteration.

\section{Performance Analysis of the Frequency Do- main Block LMS Adaptive Echo Cancel- lation System}

Figure 10 and Figure 11 show the performance analysis of the frequency-domain block LMS adaptive echo interference cancellation system when the signal to interference ratio is -10 . Before interference cancellation, the in-band PSD curve has wider fluctuation, and the distribution of the constellation is dispersal. While after interference cancellation, the in-band PSD curve becomes more flat and the out-band attenuation exceeds $40 \mathrm{~dB}$. This indicates that the frequencydomain block LMS adaptive echo interference cancellation system has remarkable inhibition ability to multipath echo interference.

\section{Conclusion}

After simulation research on six kinds of adaptive echo interference cancellation system- on the basis of LMS adaptive filtering algorithm, NLMS adaptive filtering algorithm, DLMS adaptive filtering algorithm, sign LMS adaptive filtering algorithm, frequency domain block LMS adaptive filtering algorithm, and RLS adaptive filtering algorithm, and the comparison between the performance of the effects of cancellation, convergence rate and the usage of resource, this paper selected NLMS adaptive filtering algorithm and
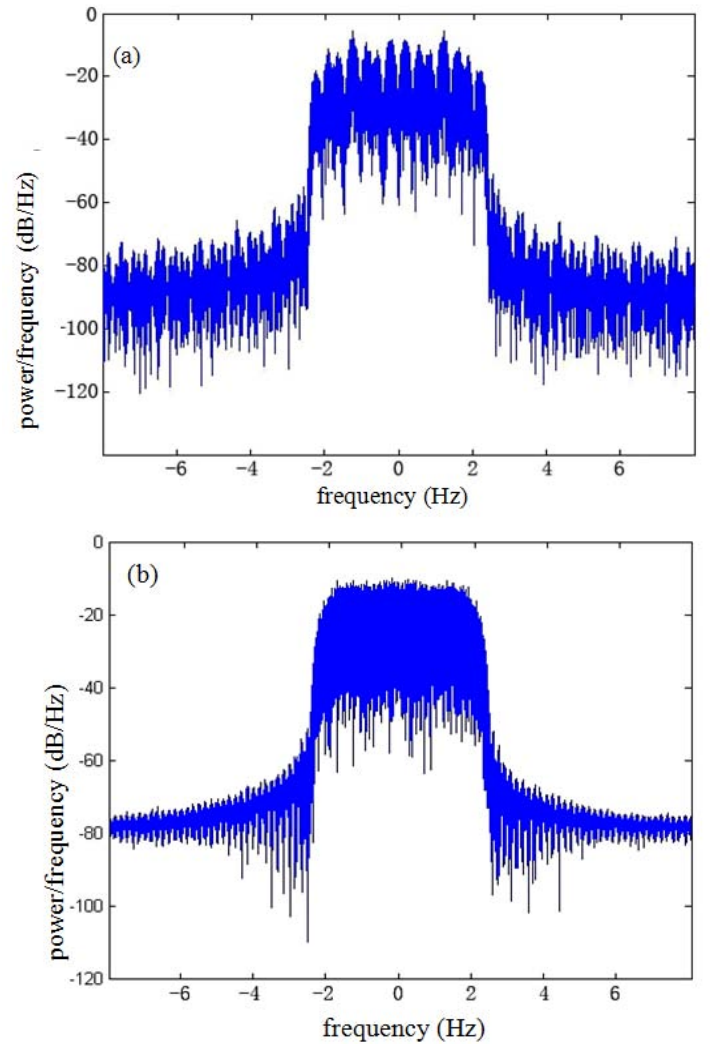

Figure 10 The comparison between the PSD curve before and after the interference cancellation when the signal to interference ratio is -10 (a). Before the interference cancellation. (b). After the interference cancellation. 

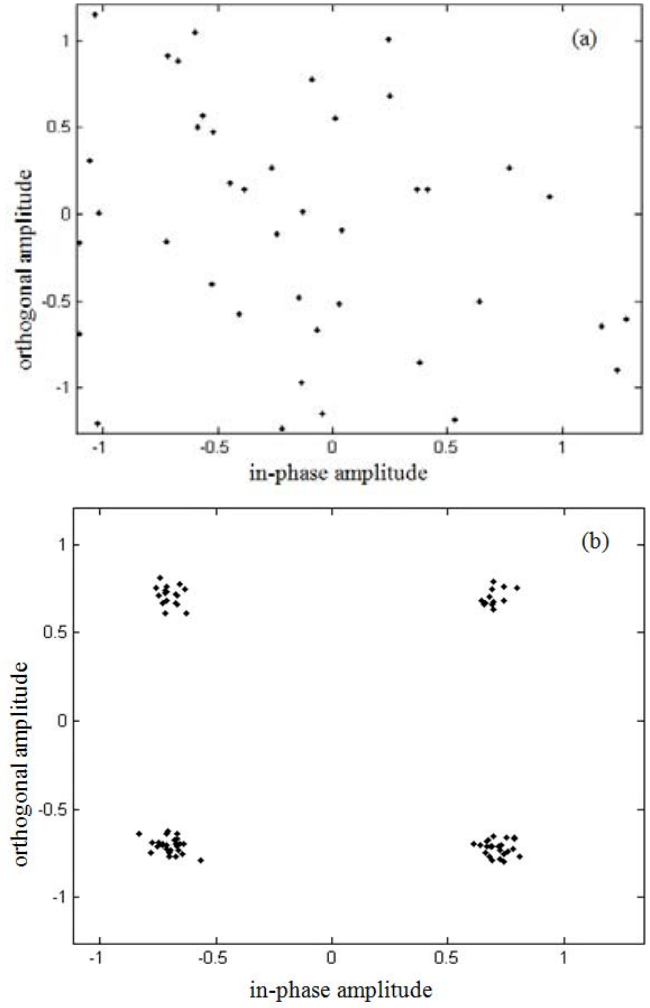

Figure 11 The comparison between the constellation before and after the interference cancellation when the signal to interference ratio is -10 . (a) Before the interference cancellation (b) After the interference cancellation

the frequency-domain block LMS algorithm for further hardware design on FPGA.

Based on the analysis and comparison of the PSD curve and the constellation of NLMS and the frequencydomain block LMS adaptive echo interference cancellation system, it concludes that the frequency-domain block LMS adaptive echo interference cancellation system has better flatness on the in-band PSD curve and better convergence effect on the constellation. Therefore, frequency-domain block LMS adaptive interference cancellation algorithm is chosen as the design with high performance-price ratio. The more points there exist, the more obvious advantages it will perform.

\section{Acknowledgments}

This work was supported by the Dr. Start Fund of Liaoning Province under project No. 20071003 and the Discipline Construction Fund of College of Information and Control Engineering of Shenyang Jianzhu University.

\section{References}

[1] Y. H. Gong, Adaptive filtering (Second Edition) timedomain adaptive filtering and smart antennas, Publishing House of Electronics Industry, BeiJing, 2003.

[2] Y. Tian, W. B. Xu, and Y. W. Zhang, Wireless FPGA Design, Publishing House of Electronics Industry, BeiJing, 2008.

[3] J.F. Meng and X.B. Hou, "The simulation and realization of LMS adaptive filter," Electronic Component \& Device Applications, Vol. 11(9), pp.67-73, 2009.

[4] M. T. Islam and Z. A. A. Rashid, "MI-NLMS adaptive beamforming algorithm for smart antenna system applications," Journal of Zhejiang University SCIENCE A, Vol.7(10), pp.1709-1716, 2006.

[5] Liu Mingchun and Li Guojin, "Research on the application of self-adaptive frequency-domain LMS algorithm on interference cancellation," World Technology Research and Development, 1:pp57-58, 2009.

[6] C. So, "Modified LMS algorithm for unbiased impulse response estimation in nonstationary noise," Electronics Letters, Vol.35(10), pp.791-792, 1999.

[7] J. Pesquet, G. Tziritas, and O. Macchi, "Modified LMS algorithms for robust ADPCM," Proc. IEEE Acoustics Speech and Signal Processing(1990), IEEE Press, Mar. pp.1405-1408, doi: 10.1109/ICASSP.1990.115652, 1990.

[8] H.C. So, "Modified LMS algorithm for unbiased impulse response estimation in nonstationary noise," Electronics Letters, Vol.35(10), pp.791-792, 1999.

[9] W. B. Chen, X. M. Geng, and W. M. Ma, "High speed adaptive DLMS algorithm and its hardware implementation," Information Technology, Vol.10, pp.5962, September, 2007.

[10] CYLIX Communication Equipment Co., Ltd, The Implementation of MATLAB and FPGA for Wireless Communication, Posts and Telecom Press, 2009.

[11] Paulo S. R. Diniz, Self-adaptive filter algorithm and implementation: Second Edition, Liu Yulin Translated. Beijing: Publishing House of Electronics Industry, 2004.

[12] Simon Haykin, Adaptive Filter Theory (Fourth Edition), Baoyu Zheng Translated, Beijing: Publishing House of Electronics Industry, 2006. 